\title{
$100 \mathrm{~kW}, 10 \mathrm{kHz}$ Medium Frequency Transformer Design Optimization and Experimental
} Verification

\author{
M. Mogorovic and D. Dujic
}

This material is posted here with permission of the IEEE. Such permission of the IEEE does not in any way imply IEEE endorsement of any of EPFL's products or services. Internal or personal use of this material is permitted. However, permission to reprint / republish this material for advertising or promotional purposes or for creating new collective works for resale or redistribution must be obtained from the IEEE by writing to pubs-permissions@ieee. org. By choosing to view this document, you agree to all provisions of the copyright laws protecting it. 


\title{
100kW, 10kHz Medium Frequency Transformer Design Optimization and Experimental Verification
}

\author{
Marko Mogorovic, Student Member, IEEE, Drazen Dujic, Senior Member, IEEE
}

\begin{abstract}
This paper describes a novel, model based, design optimization methodology for high-power medium frequency transformers for medium-voltage high-power electronic applications, namely emerging solid state transformers. Presented procedure enables the designer to interactively select the most optimal design in a simple and intuitive way, while inherently offering flexibility in terms of various design alternatives, depending on the component availability. The core of the design algorithm is explained in detail together with all of the relevant modeling and technical challenges associated with realization of a prototype. A $100 \mathrm{~kW}, 10 \mathrm{kHz}$ medium frequency transformer prototype has been designed according to the presented algorithm, practically realized and thoroughly tested in order to verify the theoretical design.
\end{abstract}

Index Terms-MFT, power electronics, modeling, design, optimization

\begin{tabular}{ll} 
& \multicolumn{1}{c}{ NomENCLATURE } \\
$U_{n}$ & Nominal primary voltage \\
$I_{n}$ & Nominal primary current \\
$f$ & Switching frequency \\
$D$ & Duty cycle \\
$L_{m}$ & Magnetization inductance \\
$L_{\sigma}$ & Total leakage inductance \\
$d_{w i c}$ & $i^{t h}$ winding to core dielectric distance \\
$d_{w i w j}$ & $i^{\text {th }}$ to $j^{\text {th }}$ winding dielectric distance \\
$d$ & Litz wire strand diameter \\
$N_{1}$ & Number of primary winding turns \\
$J$ & Current density \\
$A W G$ & Litz wire strand AWG \\
$K_{w i}$ & i-th winding height to width proportion \\
$K_{c}$ & Core cross-section proportion \\
$K_{m}$ & Normalized magnetic flux density \\
$B_{s a t}$ & Saturation flux density \\
$K, \alpha, \beta$ & Steinmetz coefficients \\
$\mu_{r}$ & Relative magnetic permeability \\
$d_{a g}$ & Air-gap length \\
$A_{c}$ & Core cross-section \\
$\mathbf{Y}_{t h}$ & Thermal network admittance matrix \\
$\Delta \mathbf{T}$ & Temperature rise vector \\
$\mathbf{Q}$ & Injected heat power vector
\end{tabular}

\section{INTRODUCTION}

The research interest in high-power switch mode power supplies operating on a medium voltage (MV) has significantly increased recently both in industry and academia [1]. Thanks

The authors are with the Power Electronics Laboratory, École Polytechnique Fédérale de Lausanne (EPFL), 1015 Lausanne, Switzerland (e-mail: marko.mogorovic@epfl.ch; drazen.dujic@epfl.ch). to the advancement in power semiconductors, magnetic materials and advanced control methods, this is possible nowadays, albeit at high costs. There are several drivers behind Solid State Transformer (SST) concept, all largely dependent on the application where these conversion structures are envisioned. Reduction of volume and weight, together with improvement in efficiency are driving the research efforts in railway domain, as discussed in [2] and demonstrated with 1.2 MW prototype in [3], [4]. On the other hand, utility three-phase applications are mostly motivated by advanced control features and flexible power flow, as recently demonstrated with 0.5 MVA prototype [5].

Independently from the application, most of the SST proposals feature some kind of Input-Series Output-Parallel (ISOP) modular converter structure, utilizing multiple identical converter stages. There are two predominant converter topologies employed for this purpose: dual active bridge (DAB) and series resonant converter (SRC). Any such converter (SST) employs at least one or usually multiple medium frequency transformers (MFTs) to achieve galvanic isolation and inputoutput voltage matching. Thus, next to the advanced control methods and novel semiconductor devices, medium-voltage high-power MFTs are one of the key enabling technologies for the future SST based power systems. The main benefit, at least from the power density point of view, is potentially substantial decrease in transformer size, enabled by fast switching of power semiconductors.

Nevertheless, design of MFTs is tied to many additional challenges compared to their traditional and technologically mature counterparts, line frequency transformers (LFTs). While LFTs operate on low grid frequency, MFTs typically operate with square voltages and non-sinusoidal currents on higher frequencies, characteristic for mentioned power electronic converters. This has an impact on the core [6]-[8] and winding [9]-[19] losses as well as on the insulation coordination, which still requires future research. Moreover, proper design of the MFT electric parameters, magnetization and leakage inductance, is paramount for the correct operation of the converter, especially in case of SRC based topologies where the MFT is a part of a resonant circuit. Therefore, the modeling and manufacturing margins associated with parameter control are more strict [20]. Furthermore, the desired increase of transformer power density is tied to the increase of the heat loss density, resulting in higher temperature gradients unless correctly addressed. Considering the increased presence of insulation media, detailed thermal modeling and coordination is required to account for these, more complicated, thermal conditions [21]. This leads to design trade-offs that 
must be correctly addressed.

Taking into account all of these considerations and associated limitations, as well as various design choices available to a designer, presents a very complex problem. Several examples of computer based design optimization can be found in the literature resulting in next MFT prototypes: litz wire/metglas $/ 25 \mathrm{~kW} / 2 \mathrm{kHz}$ [22], litz wire/nanocrystalline and ferrite/166 kW/20 kHz [23], litz wire/nanocrystalline and ferrite $/ 50 \mathrm{~kW} / 5 \mathrm{kHz}$ [24]. They are mostly based on selection and appropriate application of the earlier mentioned and often quite old models thus limiting the quality of the overall optimization process and its results. Furthermore, it is hard to compare their effectiveness and resulting prototypes due to the variety of different design choices, materials and more importantly the lack of any information regarding the dielectric withstand (e.g. PD and BIL tests). In other words, in contrast to LFTs, MFTs are still not a mature technology - there are still no general guidelines, dominant designs or standards of any kind. Improvements in terms of modeling, insulation and thermal coordination, electrical parameter control and overall design optimization are necessary in order to gain the full overview of the design possibilities and decrease the uncertainty gap between the estimations and reality. Equally important, proper testing is required to increase the experience and generate confidence in this technology thus facilitating its transition to the next step - industrial applications.

Compared to the state of the art, [22]-[24], this work introduces two novel analytical models, providing improved accuracy of hot-spot temperature rise [21] and leakage inductance [20] estimation without added computational cost. Moreover, in contrast to direct brute force approach, as used in [23], [24], the presented optimization algorithm itself uses a set of heuristics allowing pre-detection and avoidance of infeasible design domains, similar to branch and bound logic, thus saving the processing power and allowing to test much larger and more dense optimization variable sets. The proposed algorithm allows to minimize the sub-optimality gap between the selected mathematically optimal and standard design utilizing available off-the-shelf components, compared to direct selection of the most similar standard design [23], [24]. The concept of performance filtering is introduced, allowing easy and intuitive selection of the design that best matches the desired characteristics and safety margins while inherently offering flexibility in terms of multitude of alternative designs. Finally, the whole design methodology was tested in a case study of an MFT with the most challenging requirements in terms of electrical parameter accuracy and robustness to models with high modeling uncertainty such as natural air convection.

This paper is organized as follows: Section II briefly present relevant underlying models, describing relevant physics associated with MFT design, which are needed for design optimization algorithm. Section III introduces the design optimization algorithm, discusses the main design considerations and provides a complete MFT design optimization procedure while taking into account previously described modeling. The developed $100 \mathrm{~kW}, 10 \mathrm{kHz}$ MFT prototype is presented in Section IV, while experimental results related to electric, dielectric and thermal performances are presented in Section V. Finally, Section VI provides a discussion and summary of main findings.

\section{MFT MODELING}

Any optimization relies on some form of mathematical description of the relevant underlying physics. While FEM could be used to provide detailed and fairly precise modeling framework, its computational cost is often too high and convergence issues may occur when dealing with complex geometries featuring coupled multiphysics. Thus, analytical models, offering a good trade-off between accuracy and computational cost, are preferred when it comes to the overall optimization of a complex system.

This chapter provides a brief overview of analytical models, covering all relevant phenomena governing the electrical and thermal behavior of the MFT, allowing fast and numerically stable subsequent execution necessary for any design optimization approach.

\section{A. Core Losses}

Depending on the application, there are several different approaches to core loss estimation. The most relevant class of models for transformer design, recurring in the literature [6], [7], is based on the original Steinmetz equation.

$$
P_{c}=K f^{\alpha} B_{m}^{\beta}
$$

Due to the non sinusoidal waveforms, characteristic for the given applications, as shown in Fig. 1, the estimation of core loss density is done using the improved generalized Steinmetz equation IGSE [6]

$$
P_{c}=\frac{1}{T} \int_{0}^{T} k_{i}\left|\frac{\mathrm{d} B(t)}{\mathrm{d} t}\right|^{\alpha}(\Delta B)^{\beta-\alpha} \mathrm{d} t
$$

where

$$
k_{i}=\frac{K}{(2 \pi)^{\alpha-1} \int_{0}^{2 \pi}|\cos (\theta)|^{\alpha} 2^{\beta-\alpha} \mathrm{d} \theta}
$$

which, according to [22], represents the most accurate generalization of the original Steinmetz equation to an arbitrary waveform without using any additional parameters other than three Steinmetz coefficients $(K, \alpha, \beta)$.

Application of (2) on the characteristic waveform from Fig. 1, that can be analytically described with

$$
\left|\frac{\mathrm{d} B(t)}{\mathrm{d} t}\right|=\left\{\begin{array}{cl}
0 & \text { for }(1-D) T \\
\frac{2 \Delta B}{D T} & \text { for } D T
\end{array}\right.
$$

results in a closed-form analytic expression

$$
P_{c}=2^{\alpha+\beta} k_{i} f^{\alpha} B_{m}^{\beta} D^{1-\alpha}
$$

where (3), containing a numeric integral, can be curve fitted with error less than $0.15 \%$ within the relevant $\alpha$ range, as given in (6).

$$
k_{i}=\frac{K}{2^{\beta-1} \pi^{\alpha-1}\left(0.2761+\frac{1.7061}{\alpha+1.354}\right)}
$$

Finally, a simple analytical, computationally non-intensive, core loss density model is defined with (5) and (6), for the 


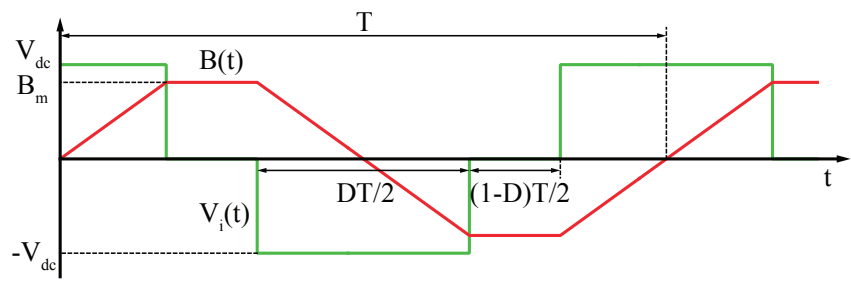

Fig. 1. Characteristic flux density waveform caused by generic three-level voltage waveform generated by converter

waveform family of interest, allowing fast execution while only using the available data sheet information.

\section{B. Winding Losses and Leakage Inductance}

Calculation of the frequency dependent winding losses and leakage inductance is based on the developed hybrid model [20], which is a generalization of the original Dowell's model [25]. Developed model is capable of properly taking into account both frequency and geometry effects characteristic for the given frequency range and increased clearance distances of high power MFTs, necessary to properly accommodate the high dielectric withstand requirements for MV applications.

According to the foil winding equivalence, as displayed in Fig. 2, the losses in each equivalent foil winding can be calculated as:

$$
P_{\sigma}=I^{2} \frac{L_{w}}{\delta \sigma^{\prime} H_{w}} m\left[\varsigma_{1}+\frac{2}{3}\left(m^{2}-1\right) \varsigma_{2}\right]
$$

where skin and proximity factors are defined with (8) and (9), respectively

$$
\begin{gathered}
\varsigma_{1}=\frac{\sinh (2 \Delta)+\sin (2 \Delta)}{\cosh (2 \Delta)-\cos (2 \Delta)} \\
\varsigma_{2}=\frac{\sinh (\Delta)-\sin (\Delta)}{\cosh (\Delta)+\cos (\Delta)}
\end{gathered}
$$

and equivalent penetration ratio and skin depth are given in (10) and (11), respectively

$$
\begin{gathered}
\Delta=\frac{d_{e q}}{\delta} \\
\delta=\sqrt{\frac{1}{\pi \mu_{0} \sigma^{\prime} f}}
\end{gathered}
$$

where the equivalent conductivity, ensuring the same DC conductivity of the foil winding equivalence can be calculated with (12)

$$
\sigma^{\prime}=\sigma \eta
$$

where the porosity factor and the equivalent foil width are calculated based on the winding geometry, as given in (13) and (14), respectively.

$$
\begin{aligned}
& \eta=\frac{N_{s v} d_{e q}}{H_{w}} \\
& d_{e q}=d \sqrt{\frac{\pi}{4}}
\end{aligned}
$$

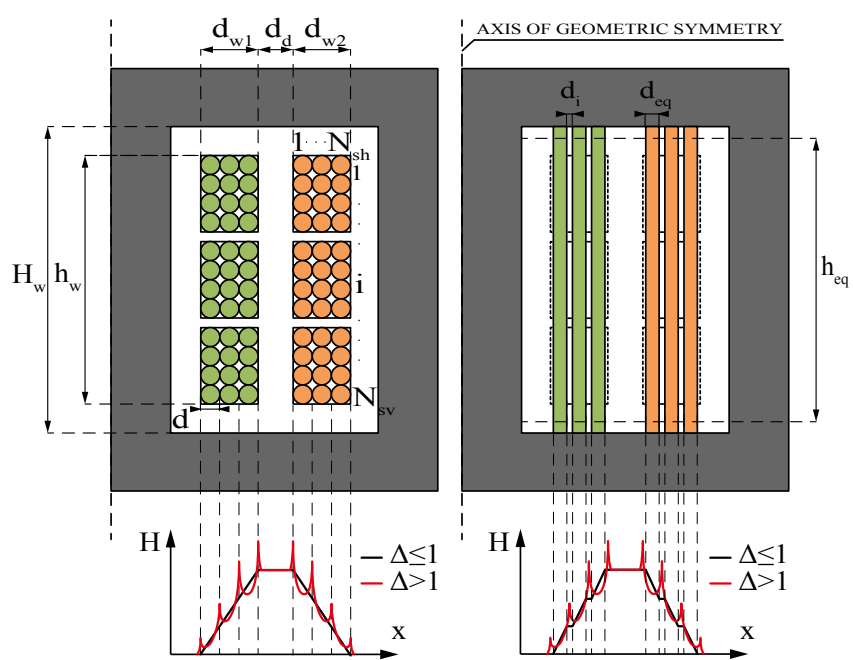

Fig. 2. Winding equivalence between round conductors and foil winding used for leakage inductance calculation

Leakage inductance can be calculated based on magnetic energy distribution within the core window area as given in (15).

$$
\begin{aligned}
& L_{\sigma}=N_{1}^{2} \mu_{0} \frac{l_{w}}{H_{w}}[\underbrace{\frac{d_{w 1 e q} m_{w 1}}{3} F_{w 1}+\frac{d_{w 2 e q} m_{w 2}}{3} F_{w 2}}_{\begin{array}{c}
\text { Frequency dependent portion due to the magnetic } \\
\text { energy within the copper volume of the windings }
\end{array}} \\
& +\underbrace{d_{d}}_{\text {Portion due to magnetic energy within }} \\
& \text { the inter-winding dielectric volume } \\
& +\underbrace{d_{w 1 i} \frac{\left(m_{w 1}-1\right)\left(2 m_{w 1}-1\right)}{6 m_{w 1}}} \\
& \text { Portion due to magnetic energy within the } \\
& \text { inter-layer dielectric of the primary winding } \\
& +\underbrace{d_{w 2 i} \frac{\left(m_{w 2}-1\right)\left(2 m_{w 2}-1\right)}{6 m_{w 2}}}_{\text {Portion due to magnetic energy within the }}] \\
& \text { inter-layer dielectric of the secondary winding }
\end{aligned}
$$

The frequency dependent portion of the leakage magnetic field, within the copper volume of the primary and secondary winding, is taken into account via Dowell's frequency dependent factor (16), applied on the equivalent foil winding as shown in Fig. 2.

$$
F_{w}=\frac{1}{2 m^{2} \Delta}\left[\left(4 m^{2}-1\right) \varphi_{1}-2\left(m^{2}-1\right) \varphi_{2}\right]
$$

where

$$
\varphi_{1}=\frac{\sinh (2 \Delta)-\sin (2 \Delta)}{\cosh (2 \Delta)-\cos (2 \Delta)}
$$

and

$$
\varphi_{2}=\frac{\sinh (\Delta)-\sin (\Delta)}{\cosh (\Delta)-\cos (\Delta)}
$$

According to [20], the equivalent length of the leakage flux path through the air is, in general, not equal to the core window height, as assumed by the original Dowell's geometry equivalence. As can be seen in Fig. 3, showing a range of winding to core window height proportions, its length is a 


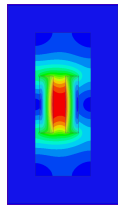

(a)

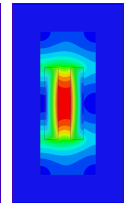

(b)

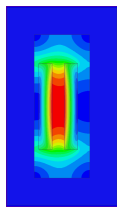

(c)

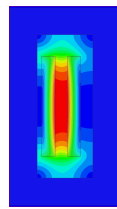

(d)

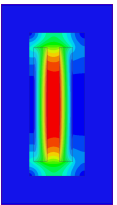

(e) (f)

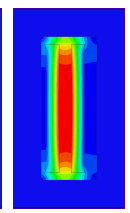

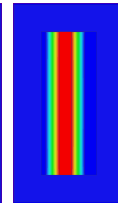

(g)
Fig. 3. 2D magnetostatic FEM simulations of the $\mathrm{H}$ field within the crosssection of the core window area for different winding heights in range from $40 \%$ (a) to $100 \%$ (g) of core window height, illustrating spacing requirements due to insulation coordination

Leakage Inductance $[\mu \mathrm{H}]$

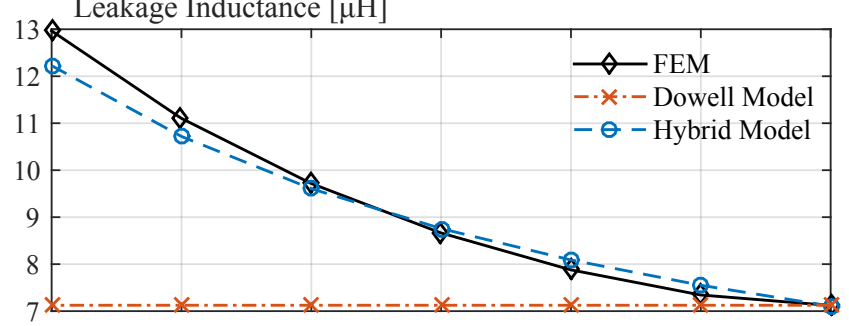

Estimation Error [\%]

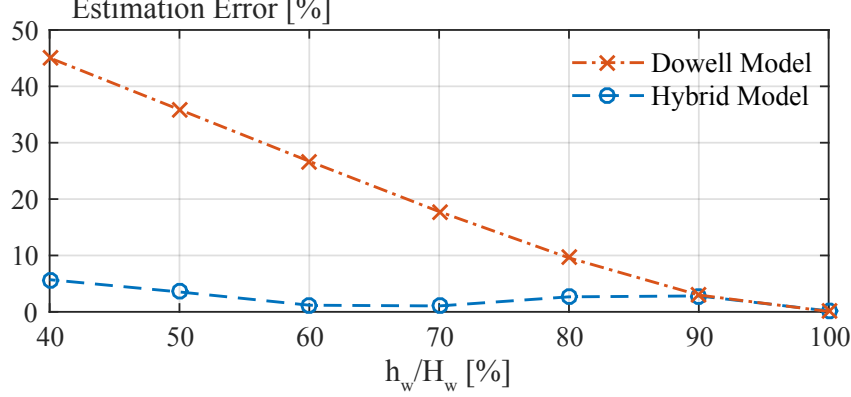

Fig. 4. Top plot: Magnetostatic FEM simulation leakage inductance estimation (black), estimation with hybrid model at zero frequency (blue dashed line), estimation with Dowell model at zero frequency (red dash-dot line); Bottom plot: DC leakage inductance estimation error of the Hybrid (blue dashed line) and Dowell (red dash-dot line) model, referred to the FEM results

function of the winding geometry. The corrected value, that properly takes into account this dependency, can be calculated with

$$
h_{e q}=\frac{h_{w}}{K_{R}}
$$

where

$$
K_{R}=1-\frac{1-e^{-\pi h_{w} /\left(d_{w 1}+d_{d}+d_{w 2}\right)}}{\pi h_{w} /\left(d_{w 1}+d_{d}+d_{w 2}\right)}
$$

is the Rogowski factor, that takes into account the uneven geometric magnetic field distribution by correcting the equivalent length (winding height) of the magnetic flux path through the air, well known for its use in the traditional LFT design [26].

As shown in [20], the modeling correction is performed by a simple substitution of $H_{w}$ with $h_{e q}$, from (19), within (7), (13) and (15) resulting in accuracy of above $94 \%$ over the entire geometry range, as shown in Fig. 4. As can be seen, in contrast to direct application of Dowel's model, this generalization allows to properly take into account the insulation spacing, thus facilitating accurate estimation for MV and HV MFT designs whose winding to core window height proportions can be rather low, depending on the dielectric properties of the utilized insulation material.

\section{Magnetizing Inductance}

For the given applications, the air gap is typically very small as the required magnetizing inductance is usually high. In our example, precise design of the magnetizing inductance (being a part of resonant tank) allow to set the desired turn-off current of resonant converter.

Therefore, a standard core reluctance model

$$
L_{m}=\frac{\mu_{0} N_{1}^{2} A_{c}}{\frac{l_{m}}{\mu_{r}}+d_{a g}}
$$

supplemented with a simple empirical air-gap fringing flux model [27], sufficiently accurate for small air-gaps

$$
L_{m}^{\prime}=L_{m} F_{F R}
$$

where

$$
F_{F R}=1+\frac{d_{a g}}{\sqrt{A_{c}}} \ln \left(\frac{2 H_{w}}{d_{a g}}\right)
$$

is used for the estimation of the magnetizing inductance.

Taking into account the variations of the core characteristics due to the manufacturing imperfections, together with the limited accuracy of the transformer assembly process, implementation of a more accurate and accordingly more complicated model was judged as unnecessary for design optimization.

\section{Thermal Modeling}

The thermal modeling is based on the detailed steadystate thermal network model presented in [21], consisting of conduction, convection and radiation thermal resistances, as summarized in Table I. It is constructed based on the 3D transformer geometry using its symmetry, as illustrated in Fig. 5. Even though the thermal network is derived based on one 2D symetry detail, it takes into account the different cooling conditions of both portions of the windings, inside and outside of the core window area. As can be seen from Fig. 6, due to the very high thermal conductivity of copper, the temperature along the winding path is almost constant allowing to account for the two different cooling environments along the winding path by simply connecting the corresponding thermal resistances in parallel, each depicted directly as a single resistance in Fig. 5b. The resulting thermal network, based on merging of the two MFT symmetry details, provides a very good hot-spot temperature estimation of a real 3D system, as shown in Table II, without increasing the modeling complexity.

The admittance matrix is generated based on the thermal network, allowing efficient temperature calculation, according to the injected power of winding and core losses, where each temperature estimation corresponds to the inversion of the generated admittance matrix according to (24).

$$
\mathbf{Q}_{(n)}=\mathbf{Y}_{t h_{\left(n_{x} n\right)}} \Delta \mathbf{T}_{(n)}
$$

The computational cost of the model is further reduced by applying the Kron reduction [31] to eliminate the nodes with 

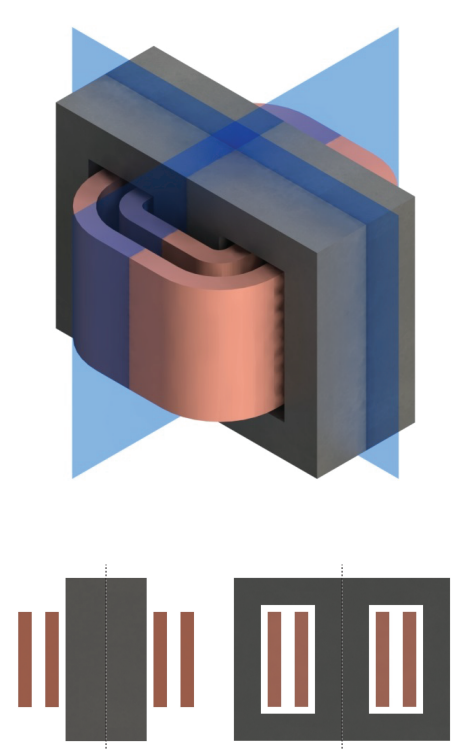

(a)

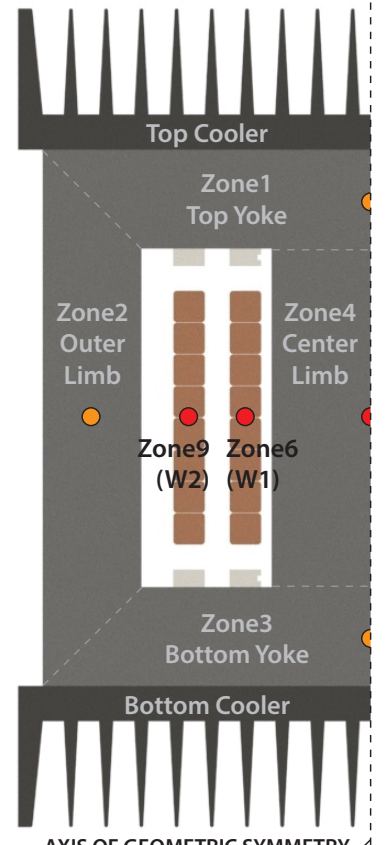

AXIS OF GEOMETRIC SYMMETRY

Fig. 5. (a) Geometric symmetry of the MFT in respect to heat transfer and partitioning of the 2D symmetry detail into zones; (b) Detailed steady state thermal network model of the MFT based on conduction, convection and radiation thermal resistances (nodes without heat injection - gray, expected hot-spot nodes for the given design - red)

TABLE I: Heat transfer mechanisms utilised for thermal modeling purposes

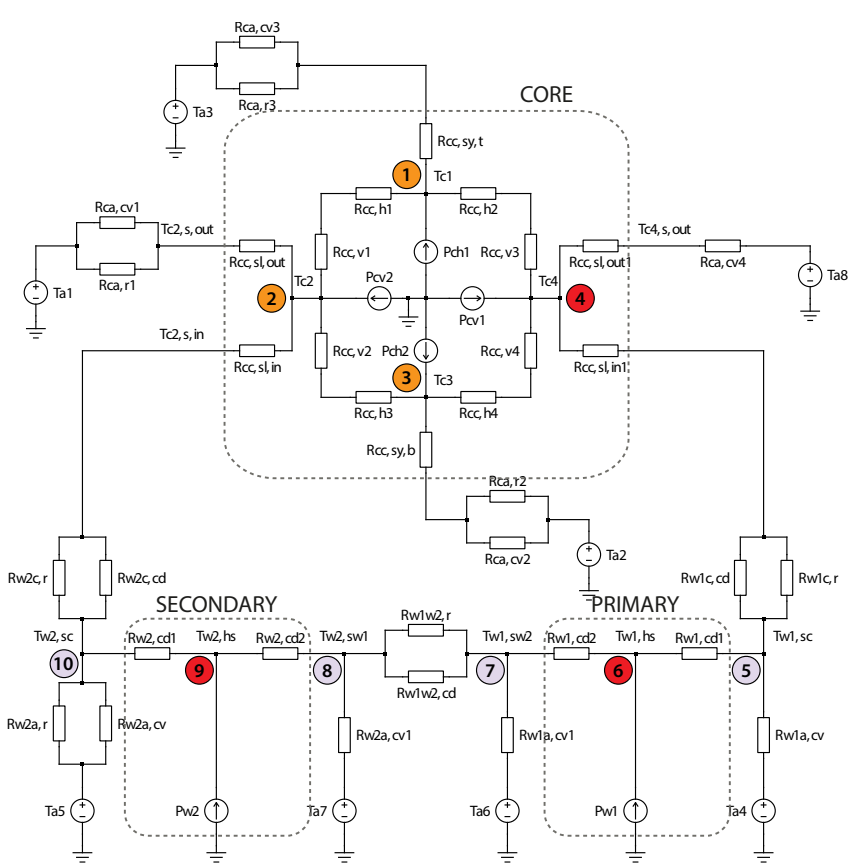

(b)

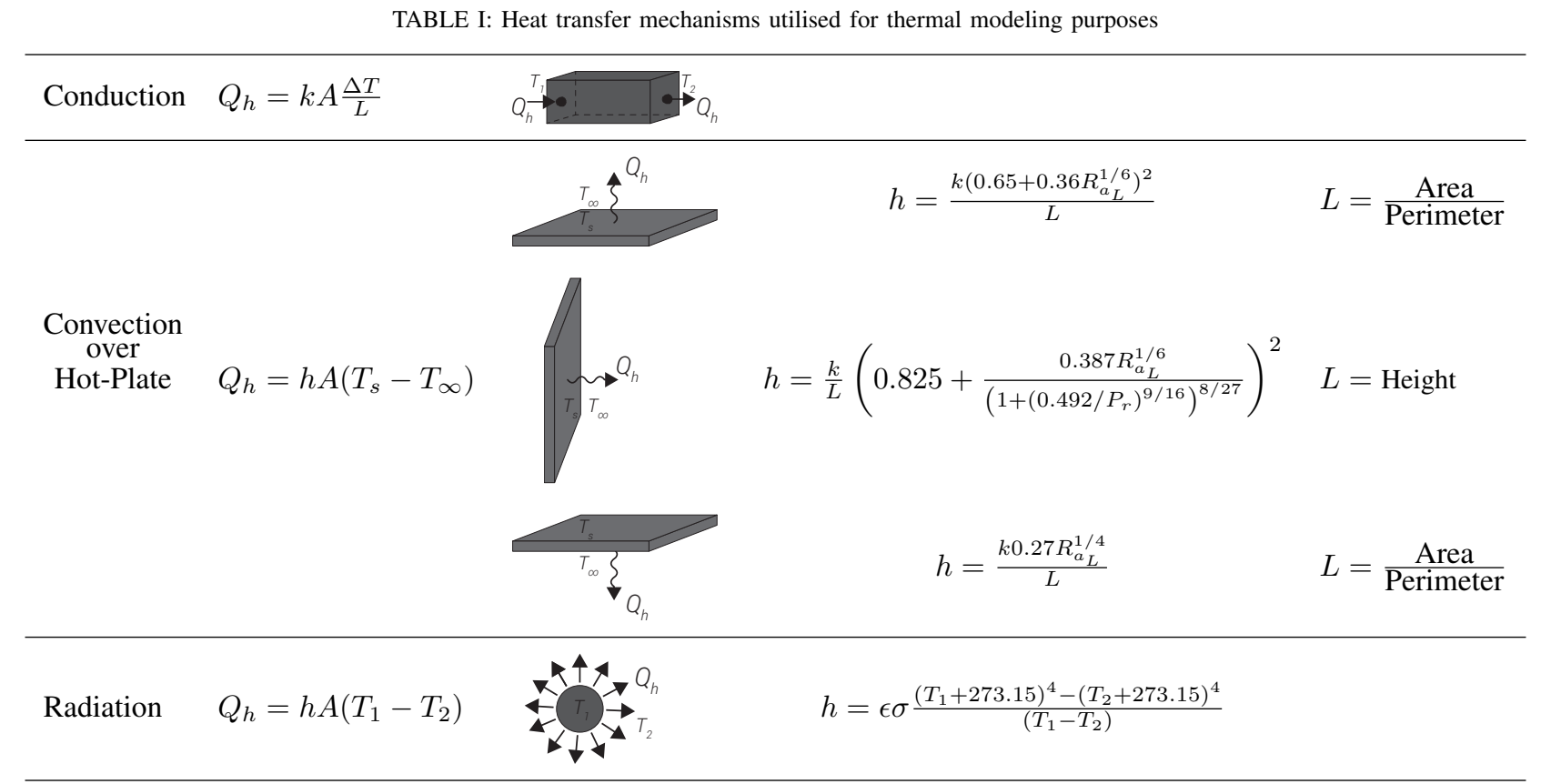

where: $R_{a_{L}}$ - Rayleigh number, $P_{r}$ - Prandtl number, $\epsilon$ - Emissivity, $\sigma$ - Stefan-Boltzmann constant [28]-[30]

zero power injection, thus reducing the admittance matrix of the thermal network, given in Fig. 5b, from (10x10) to $(6 \times 6)$ dimension as shown in (25) and (26).

$$
\left[\begin{array}{c}
\mathbf{Q}_{A_{(m)}} \\
\mathbf{0}_{(p)}
\end{array}\right]=\left[\begin{array}{ll}
\mathbf{Y}_{A A_{\left(m_{x} m\right)}} & \mathbf{Y}_{A B_{\left(m_{x} p\right)}} \\
\mathbf{Y}_{B A_{\left(p_{x} m\right)}} & \mathbf{Y}_{B B_{\left(p_{x} p\right)}}
\end{array}\right]\left[\begin{array}{c}
\Delta \mathbf{T}_{A_{(m)}} \\
\Delta \mathbf{T}_{B_{(p)}}
\end{array}\right]
$$

$$
\begin{aligned}
& \Delta \mathbf{T}_{A_{(m)}}= \\
& =\left(\mathbf{Y}_{A A_{\left(m_{x} m\right)}}-\mathbf{Y}_{A B_{\left(m_{x} p\right)}} \mathbf{Y}_{B B_{\left(p_{x} p\right)}}^{-1} \mathbf{Y}_{B A_{\left(p_{x} m\right)}}\right)^{-1} \mathbf{Q}_{A_{(m)}} \\
& =\mathbf{Y}_{\operatorname{Kron}_{\left(m_{x} m\right)}}^{-1} \mathbf{Q}_{A_{(m)}}
\end{aligned}
$$

The derived thermal network structure is made general so 


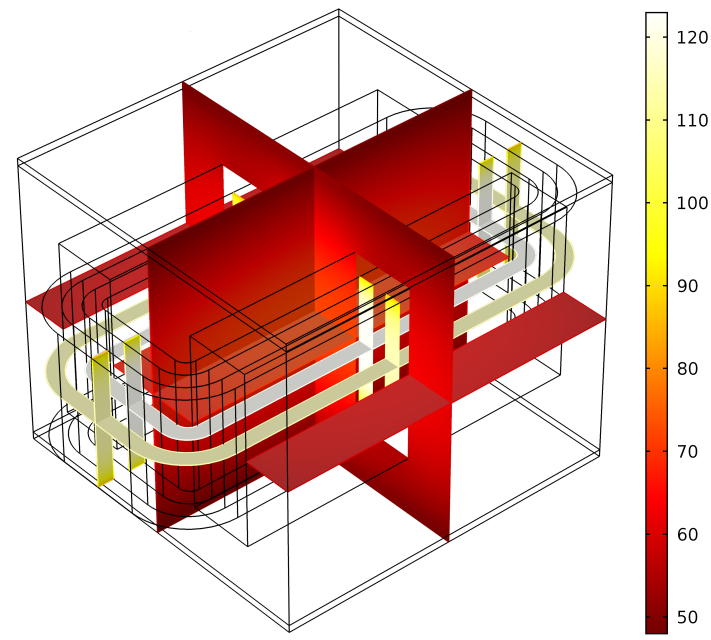

Fig. 6. 3D FEM thermal MFT simulation taking into account the anisotropic thermal conductivity of the windings

TABLE II: Hot-spot temperature estimations

\begin{tabular}{|c|c|c|c|}
\hline Hot-spot nodes & $T_{4}\left[{ }^{\circ} \mathrm{C}\right]$ & $T_{6}\left[{ }^{\circ} \mathrm{C}\right]$ & $T_{9}\left[{ }^{\circ} \mathrm{C}\right]$ \\
\hline FEM 3D full & 75 & 122 & 113 \\
\hline Analytical & 73.75 & 124.6 & 116.3 \\
\hline Max Allowed & 100 & 150 & 150 \\
\hline
\end{tabular}

that the developed methodology can directly be applied to any different MFT design by simply changing the appropriate thermal coupling resistances. In this way, a sufficiently accurate and detailed thermal model, with acceptable computational cost for design optimization purposes, is constructed.

A design optimization algorithm, based on aforementioned models, is presented next and used to design an MFT with electrical specifications outlined in Table III.

\section{MFT DESIGN OPTIMIZATION}

There exists a multitude of formal mathematical optimization tools depending on the problem characteristics. The problem at hand is non-linear, non-convex and comprises a mix of continuous and discrete variables which classifies it as difficult and, in general, it is not possible to formally guarantee convergence or that the found solution is in fact the global optimum. Further more, proper cost setting of the multivariable objective function, in order to reflect the desired design characteristics, is not straightforward or intuitive and any adjustments require re-optimization. The method of choice, described in this paper, relays on brute-force, organized using certain heuristics in such a way to allow efficient detection and avoidance of the possible design domain portions that will for sure be infeasible, similar to branch and bound logic. This greatly improves the performance of this, normally very computationally intensive, approach while preserving the inherent reliability.

The core of the proposed MFT design optimization method is the brute-force algorithm displayed in Fig. 7. It combines the functionality from design optimization techniques described

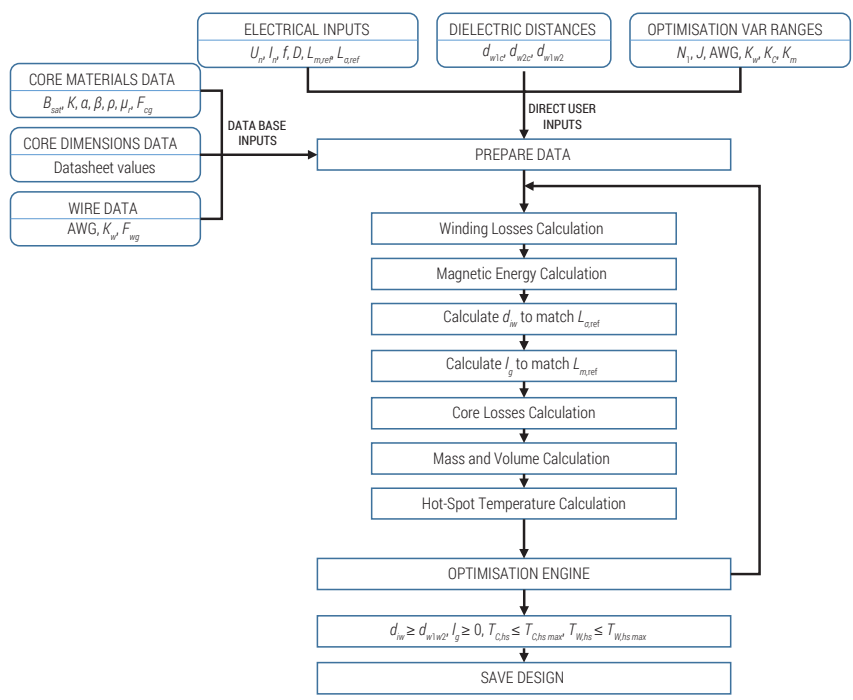

Fig. 7. Model based brute force MFT design optimization algorithm

TABLE III: MFT prototype reference electric specifications

\begin{tabular}{|ll|ll|ll|}
\hline$P_{n}$ & $100 \mathrm{~kW}$ & $V_{1}$ & $750 \mathrm{~V}$ & $L_{\sigma 1}, L_{\sigma 2}$ & $3.3 \mu \mathrm{H}$ \\
$f_{s w}$ & $10 \mathrm{kHz}$ & $V_{2}$ & $750 \mathrm{~V}$ & $L_{m}$ & $750 \mu \mathrm{H}$ \\
\hline
\end{tabular}

in [22]-[24] and offers additional degrees of freedom (e.g. different characteristics and geometric proportions of primary and secondary winding), thus generating a much more versatile design population and uncovering more design possibilities. This is enabled by proper transformations and use of novel sophisticated analytic models [20], [21] providing improved accuracy and lower computational cost, both paramount for the quality of the overall optimization.

All the inputs are classified in two groups: database inputs - containing all the relevant component data sheet specifications and direct user inputs - electric and dielectric parameter references and optimization variables ranges (primary number of turns, current and flux densities, litz wire strand AWGs and normalized geometrical proportions). The function "prepare data" generates the optimization space where each variation of optimization variables corresponds to a single fully defined potential MFT design. Each of these designs is processed, determining its characteristics and feasibility.

According to described and improved analytical models, winding losses and magnetic energy density distribution are calculated. The dielectric clearance between the windings is reverse calculated to match the reference leakage inductance, based on the magnetic energy density calculation. Air gap length is calculated to match the magnetization inductance reference. The mass and the volume are calculated based on the design specifications and material properties. The core loss density is calculated and the hot-spot temperatures are estimated with the described custom made detailed thermal network model that offers an optimal trade-off between the accuracy and computation speed.

Based on these results and using specific heuristics, the "optimization engine" function guides the design selection process, detecting infeasible design space domains and avoid- 
ing wasting computational power on their processing. Finally, all the designs that pass the feasibility check are saved into a design database for post processing.

The proposed optimization methodology consists of two steps. In the first step, all optimization variables are varied freely, in a mathematically arbitrary way, thus exposing all mathematically feasible designs, as shown in Fig. 8 with circle markers, where the color-code corresponds to the highest hotspot temperature within the windings (calculated during evaluation of that particular design). These designs are interactively filtered in terms of key performance indicators (maximum weight, volume and hot-spot temperatures, efficiency etc.) allowing to arbitrarily shift and narrow down the filtered design domain by relaxing and tightening the corresponding constraints, as highlighted with black star markers. This feature assume expert user with knowledge of acceptable tradeoffs for a given application.

In the second step, the filtered designs are analyzed against available standard core and wire geometries and the design is re-optimized with fixed discrete values corresponding to selected most similar components, as displayed in Fig. 8 with red star markers. These designs are filtered in a same way as before, using the interactively defined performance filters, as highlighted with green star markers. The final design is selected from this set, based on various additional designer preferences, such as construction simplicity, experience etc., as highlighted with the purple star marker.

In contrast to analysis of one or multiple sets of Paretooptimal solutions, characteristic for formal optimization algorithms, this approach allows the designer to interactively select the best design trade-off in a very simple and intuitive way and provides great flexibility to choose between multiple design alternatives with very similar performance and specifications, but comprised of different components in case some are unavailable or too costly to be customized. Therefore, not only the characteristics of the MFT design itself, but also the whole design process and the prototype realization time are optimized.

With the described two step approach, the proposed method minimizes the sub-optimality gap between the theoretical and standard MFT designs, comprised of off-the-shelf components, and inherently provides a valuable insight into the positioning of the solutions utilizing specific hardware relative to all theoretically possible designs in a clear and intuitive way. This may be a very valuable input when it comes to component standardization, as it can clearly be seen what core or wire geometries are covering which MFT power, voltage and efficiency ranges.

The upper boundary of the feasibility set plots represents the Pareto front, related to the trade-off between maximum achievable efficiency and power density, as shown in Fig. 8. Its descending trend shows how efficiency must be sacrificed for the power density to increase. The lower boundary represents the thermal limit. It shows the implications of the size decrease on the cooling conditions and therefore maximum acceptable losses.

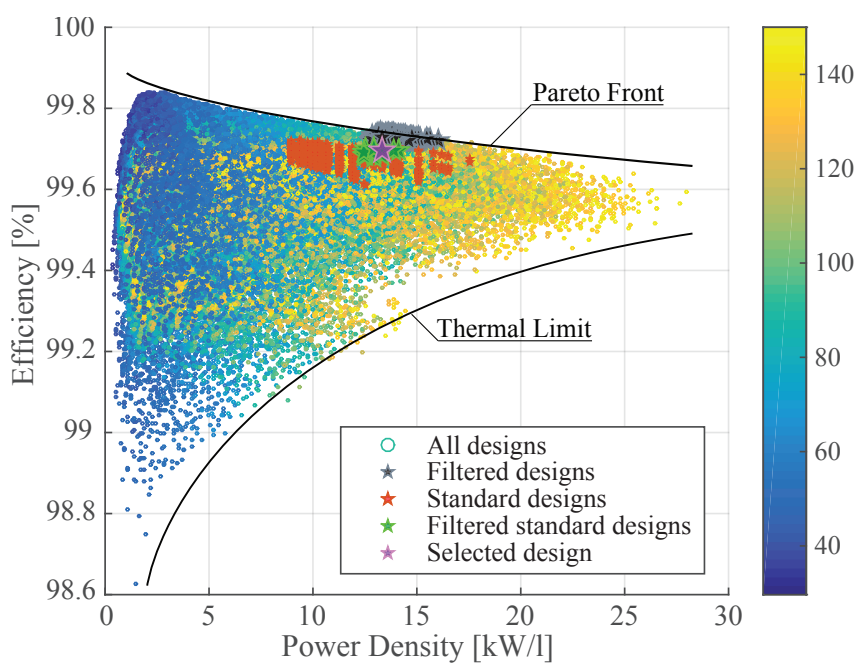

(a)

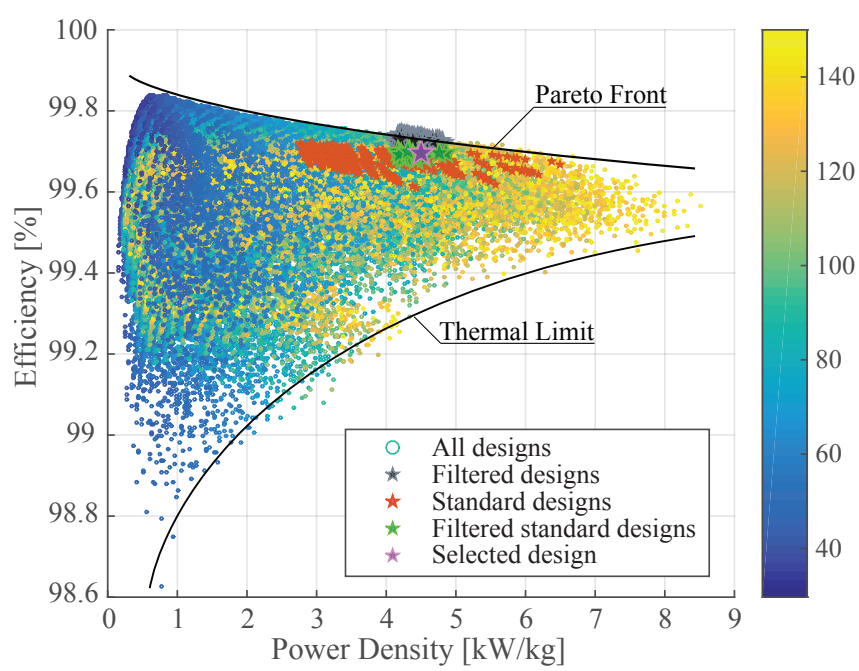

(b)

Fig. 8. Plot of all possible MFT designs (around two million designs executed in approximately three hours) and filtered optimal designs according to specifications from Table III: (a) Efficiency vs. volume power density; (b) Efficiency vs. weight power density; Color code corresponds to the highest hot-spot temperature within the windings.

\section{MFT PROTOTYPE}

Applying the described optimization process to a transformer with electric specifications shown in Table III, an optimal MFT design is selected, as displayed in Fig. 8 and marked with a star. The realized prototype is an air insulated and air cooled shell type MFT utilizing square litz wire for windings and N87 SIFERRIT U-cores.

Initially, the 3D CAD models are created in SOLIDWORKS, according to the resulting design specifications generated by the optimization algorithm, providing support to design and manufacturing process of coil formers. Primary and secondary winding each consist of eight turns of the square profiled $(8.7 \times 8.7 \pm 0.2 \mathrm{~mm})$ copper litz wire with $1400 A W G 32$ litz strands and a total copper crossection of $43.96 \mathrm{~mm}^{2}$, wound on the corresponding coil formers, as displayed in Fig. 9b. The primary and secondary coil 
formers have been produced using additive manufacturing process (3D printing) out of PA2200 high strength thermally resistant plastic. They have been designed and optimized, both to maximize the mechanical support strength and area for natural convective air cooling of the windings with respect to the mechanical properties of the selected wire, as shown in Fig. 9a. The MFT core is made of 48 (12 x 4) SIFERRITE U-cores (UU9316 - CF139).

The realized $100 \mathrm{~kW}, 10 \mathrm{kHz}$ MFT prototype with integrated resonant capacitor bank (distributed between primary and secondary winding) held by custom designed bus-bars, is displayed in Fig. 9c. Even though the prototype is almost identical as the 3D CAD design, there exist a couple of small (measured in parts of $\mathrm{mm}$ ) differences in the geometry due to the limitations of the technological assembly process and material imperfections which effect the electric parameters of the MFT.

Considering brittle nature of ferrite materials and manufacturing tolerances in terms of the surfaces and dimensions, mechanical amortization is necessary at every surface of contact with another solid material. Due to this effect, there is a significant difference between the reference magnetizing inductance from Table III and the one that was measured on the MFT prototype $\left(L_{m}=750 \mu \mathrm{H}\right)$ as the width of the interfacing pad maintaining the air-gap distance had to be chosen larger than calculated due to mentioned mechanical constraints.

On the other hand, bending of the large cross-section litz wire is not a simple task and relatively large bending radius, that is hard to achieve in the corner regions of the winding, needs to be considered in order to limit the bulging and twisting effect of the wire. Furthermore, there exists an offset in the winding placement of around $1 \mathrm{~mm}$ compared to the 3D $\mathrm{CAD}$ and the wire itself has a slightly larger profile than stated in the data sheet. Consequently, the $\mathrm{H}$ field distribution in the window area and the leakage inductance are affected, and as a result, the measured leakage inductance $\left(L_{\sigma 1}=L_{\sigma 2}=\right.$
$4.2 \mu \mathrm{H})$ is higher than the reference value from Table III. Leakage inductance is very sensitive to imprecision of winding geometry, inter-winding dielectric spacing in particular, as the magnetic energy density is the highest in this area. That is why, even these tiny mechanical imperfections, in $1 \mathrm{~mm}$ order of magnitude, can have a significant relative influence on the leakage inductance of MFTs for resonant converters where the reference value is typically very low, in a couple of $\mu \mathrm{H}$ order of magnitude depending on the rated power and voltage.

\section{EXPERIMENTAL VERIFICATION}

Several tests, under laboratory conditions, have been performed on the realized MFT prototype in order to verify design targets and operational performances.

\section{A. Electrical Parameter Measurement}

Before experimentally commissioning the MFT, its electric parameters were verified using the network vector analyzer Bode 100, as shown in Fig. 10. Plots of the measured and estimated total leakage inductance, based on the exact dimensions of the realized prototype geometry with its slight imperfections, are displayed in Fig. 11. It can be seen that the developed hybrid leakage inductance model provides a much better estimation compared to direct application of the original Dowell's model, with less than $2.5 \%$ error in the entire frequency range.

Plots of the measured and estimated total MFT series resistance are displayed in Fig. 12. It can be seen that the estimation follows the measured result quite well with relative error less than $5 \%$ at $10 \mathrm{kHz}$ and $20 \%$ in the entire frequency

TABLE IV: MFT losses at $10 \mathrm{kHz}, 100 \mathrm{~kW}$ operation

\begin{tabular}{|c|c|c|c|}
\hline & $R_{\sigma}[\Omega]$ & $P_{\sigma}[\mathrm{W}]$ & $P_{c}[\mathrm{~W}]$ \\
\hline Estimated & 4.74 & 154.2 & 150.5 \\
\hline Measured & 4.59 & 149.6 & $/$ \\
\hline
\end{tabular}

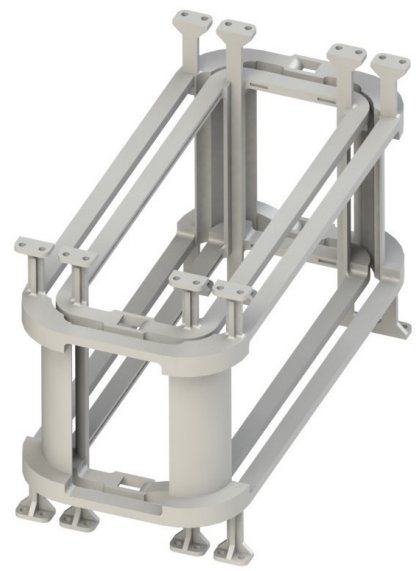

(a)

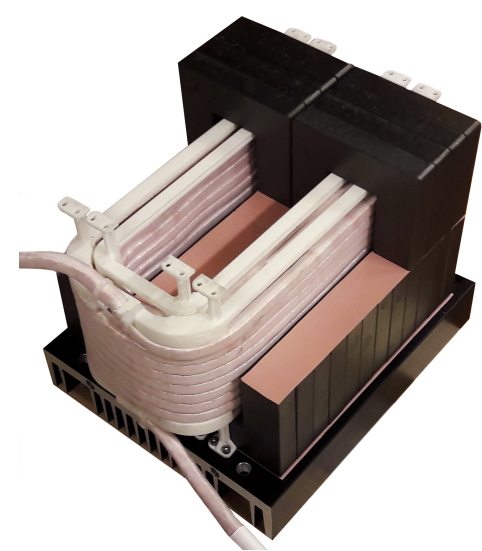

(b)

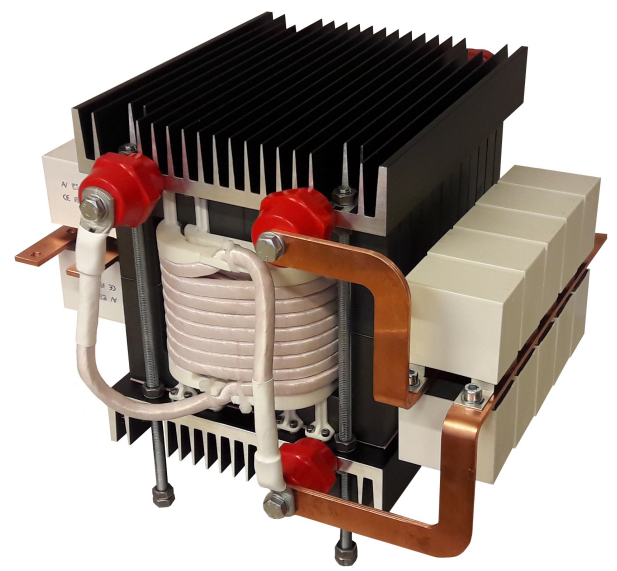

(c)

Fig. 9. Optimal design resulting from the optimization algorithm: (a) MFT 3D CAD render (b) Primary and secondary coil formers 3D CAD render (c) Realized MFT prototype. Dimensions (H/W/D) with and without the resonant capacitor bank and the bushings are 235/340/330 mm and 235/200/232 mm, respectively. 


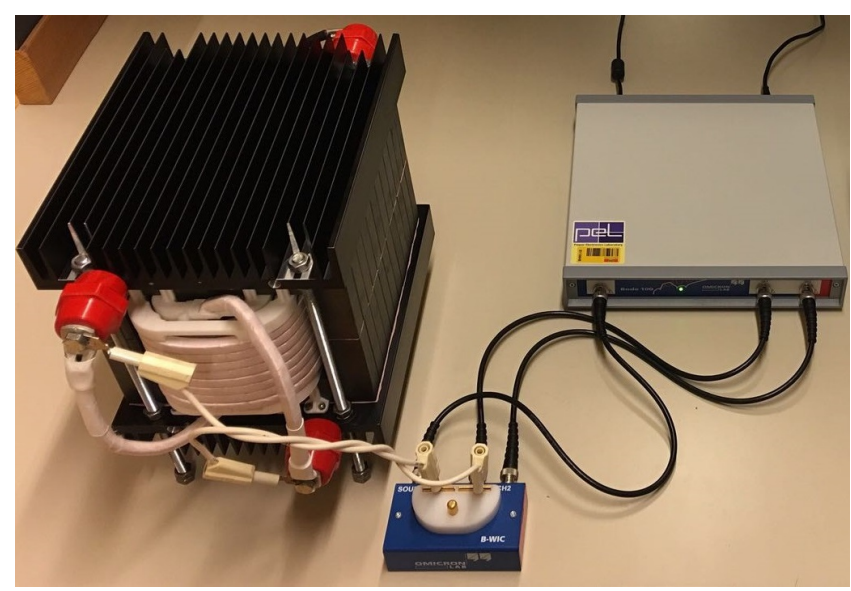

Fig. 10. Measurement of the electric parameters of the MFT

range. It is important to mention that the series resistance of the connecting cables has been measured a priory and its effect on the MFT series resistance measurement has been canceled.

The distribution of winding and core losses of the realized MFT prototype, at nominal operation $(100 \mathrm{~kW}$ at $10 \mathrm{kHz})$, are summarized in Table IV. Since, it is not possible to properly measure the MFT losses without a dedicated calorimetric setup, winding losses are calculated based on the nominal resonant current RMS and AC resistance measurement at operation frequency of $10 \mathrm{kHz}$, whereas core losses are only estimated.

\section{B. Electrical Measurements in a Resonant Test Setup}

To carry out the electrical tests on the MFT prototype, a full power rated resonant test setup has been assembled. The electrical scheme of the test setup and its physical layout inside the protective cage are shown in Fig. 13 and Fig. 14, respectively. The setup consists of two H-bridge modules, one actively switched and the other operating as a diode rectifier, with their corresponding DC-bus capacitors. The resonant tank comprised of the MFT and the distributed resonant capacitors $\left(C_{r 1}\right.$ and $\left.C_{r 2}\right)$ is inserted between two converters and two DC sources, one operated in voltage $\left(U_{D C 1}\right)$ and the other in current $\left(I_{D C}\right)$ control mode are used. Test setup is operated as a resonant converter at the constant frequency $f_{s w}=10 \mathrm{kHz}$ and with the constant duty cycle $D=0.5$. Positive ends of the DC-buses on the primary and secondary side are connected, whereas their negative ends are connected through a current source $\left(I_{D C}\right)$ thus allowing the circulation of the energy from the secondary side back to the primary side DC-bus, as displayed in Fig. 13.

Both primary $\left(C_{r 1}\right)$ and secondary $\left(C_{r 2}\right)$ capacitor of the distributed resonant capacitor bank are realized as a parallel connection of eight $(7 \times 5 \mu \mathrm{F}+1 \times 2.5 \mu \mathrm{F})$ AC film capacitors, held by bus-bars, giving a total resonant tank capacitance of $18.75 \mu \mathrm{F}$ and consequently the resonant tank frequency of $f_{0}=12.7 \mathrm{kHz}$. Therefore, the desired sub-resonant converter operation is achieved, providing soft switching turn-on for the primary side switches and zero current switching for the secondary side diodes.
Leakage Inductance $[\mu \mathrm{H}]$

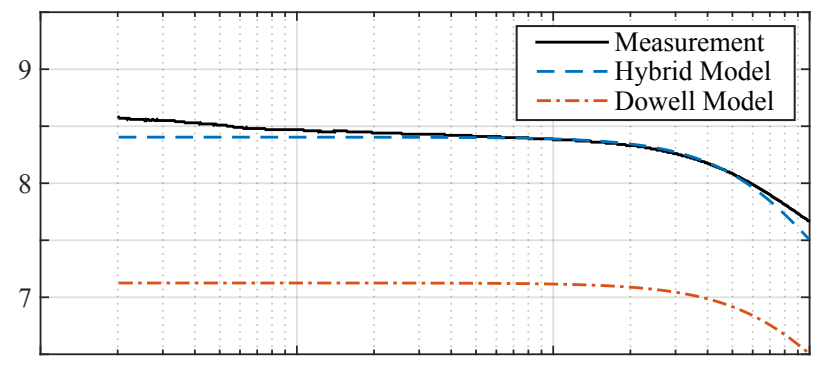

Estimation Error [\%]

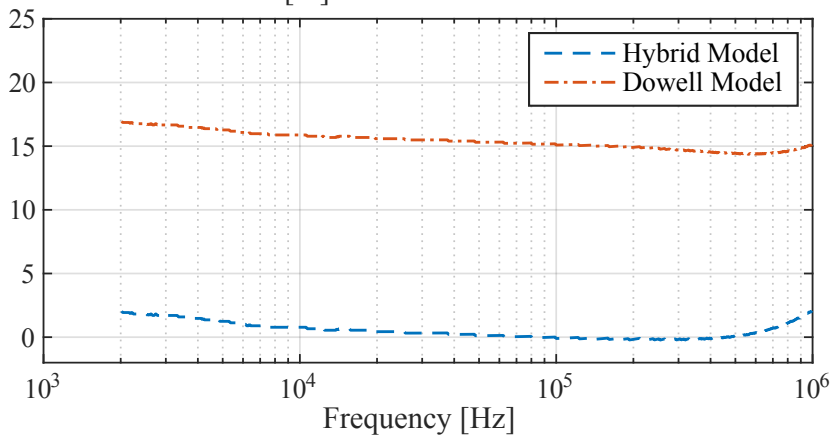

Fig. 11. Top: plots of total leakage inductance $\left(L_{t o t}=L_{\sigma 1}+L_{\sigma 2}\right)$ measurement (black), estimation with hybrid model (blue dashed line) and estimation with Dowell model (red dash-dot line); Bottom: plots of total leakage inductance estimation error of the Hybrid (blue dashed line) and Dowell (red dash-dot line) model, referred to the measurement
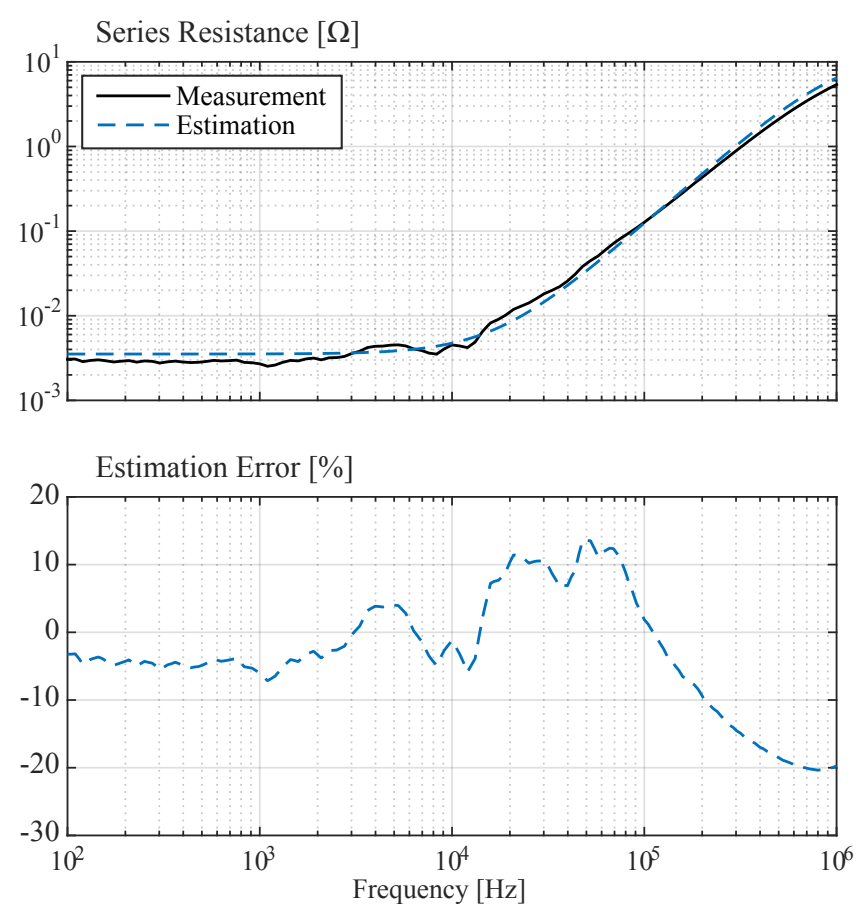

Fig. 12. Top: plots of total MFT series resistance measurement (black) and estimation (blue dashed line); Bottom: plot of total MFT series resistance estimation error, referred to the measurement

The actual H-bridges are realized by utilizing two phases of two three-phase SEMIKRON Skiip modules, as seen in Fig. 14. The primary side H-bridge is actively controlled 
with PWM signals generated by the ABB AC 800PEC high performance controller where all relevant control functions are implemented in MATLAB Simulink and executable code is generated using real-time code generation. All devices within the protective test cage, two DC sources, 8-channel oscilloscope, and AC 800PEC controller, as displayed in Fig. 14, are connected through their ethernet connection to the multi-port active switch communicating with the PC via the optical cable thus ensuring safety and complete electrical decoupling of the operator from the high power test setup.

Voltages on the AC side of the H-bridge converter $\left(U_{12}\right)$ and the diode rectifier $\left(U_{23}\right)$ are measured together with the resonant capacitor voltages on both the primary $\left(U_{C r 1}\right)$ and secondary $\left(U_{C r 2}\right)$ side, as well as the primary $\left(I_{1}\right)$ and secondary $\left(I_{2}\right)$ resonant currents. Furthermore, the DC-bus voltages on each Skiip module, and the output AC currents of the two converters are measured with additional sensors and used to implement safety features (e.g. over-voltage and overcurrent protection) within the controller software. Voltages and currents of both DC sources are monitored to evaluate the power injected into the test setup, covering the total losses during the operation.

The MFT is equipped with wireless thermocouple probes at various accessible expected hot-spots within the core and the windings for constant thermal monitoring, as displayed in Fig. 15, whereas the temperature of the outer surfaces is monitored with the thermal camera.

The measured voltage and current waveforms, from the primary and secondary side of the MFT at nominal power operation, are displayed in Fig. 16a and Fig. 16b, respectively. Top plots show the voltage waveforms on the output of the $\mathrm{H}$ Bridge $\left(U_{12}\right)$ and the input of the diode rectifier $\left(U_{34}\right)$. Primary side voltage is regulated by DC voltage source $U_{D C 1}$ to $750 \mathrm{~V}$. Middle plots show the voltages on the primary $\left(U_{C r 1}\right)$ and secondary $\left(U_{C r 2}\right)$ side resonant capacitors, while bottom plots show the primary $\left(I_{1}\right)$ and secondary $\left(I_{2}\right)$ resonant current waveforms. One can observe that resonant tank current and capacitor voltage are in quadrature, without any distortion, indicating well balanced operation. Resonant current plots show that the resonant frequency corresponds to the calculated value $\left(f_{0}\right)$ and that the soft-switching is achieved. The maximum switch-off current of the IGBTs is therefore limited by the peak value of the magnetization current $\left(I_{m_{\max }}=25 \mathrm{~A}\right)$.

Plots of the primary and secondary resonant currents, under various loading conditions ranging from $0 \%$ to $100 \%$ of the rated power, are provided in Fig. 17a and Fig. 17b, respectively. Under no-load conditions, only the magnetizing current is present in the circuit, and as the power transfer is increased, typical resonant waveform is established with rms value proportional to the operating power level. Due to inability to reduce the dead-time below the minimum $3 \mu$ s set by the gate driver, it can be observed from Fig. 16 that a minor and incomplete loss of the zero voltage switching occurs at $100 \mathrm{~kW}$ operating point. This was not a major concern during MFT testing, considering that the resonant test setup was of sufficient ratings, and the objective of the paper is not on the converter optimization.

Total losses of the whole resonant test setup can be calcu-

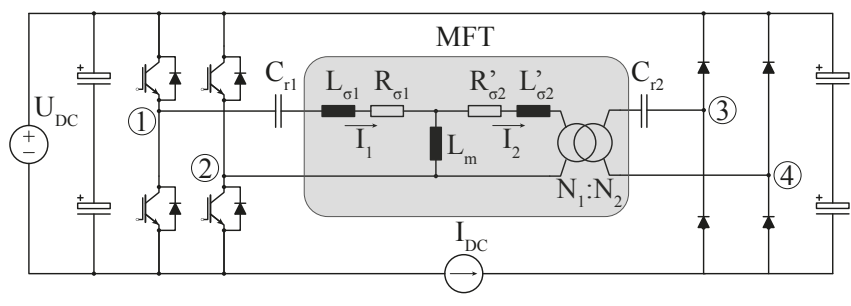

Fig. 13. Electrical scheme of the B2B resonant test setup

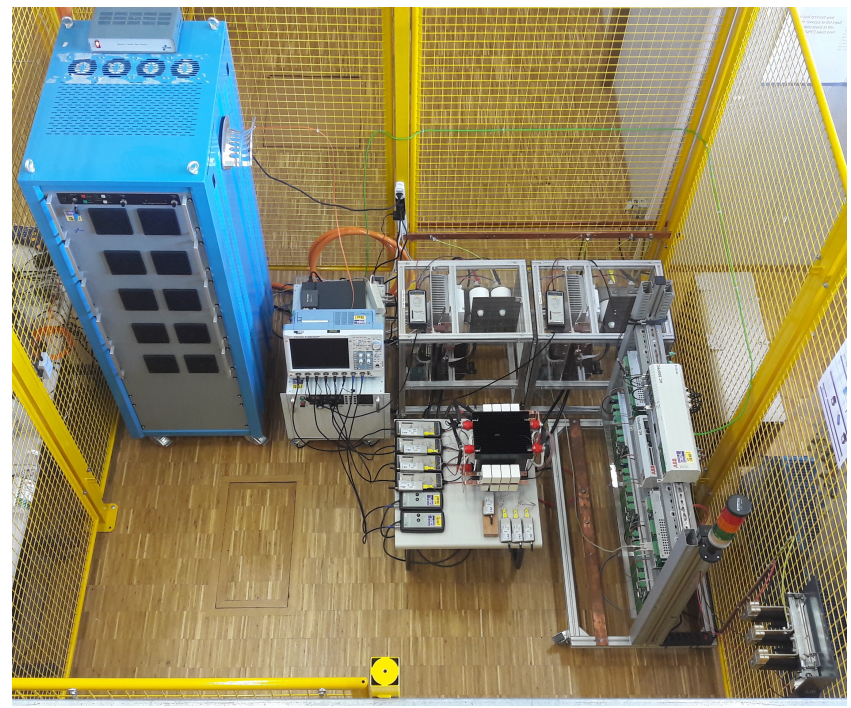

Fig. 14. Layout of the test setup within the protective cage

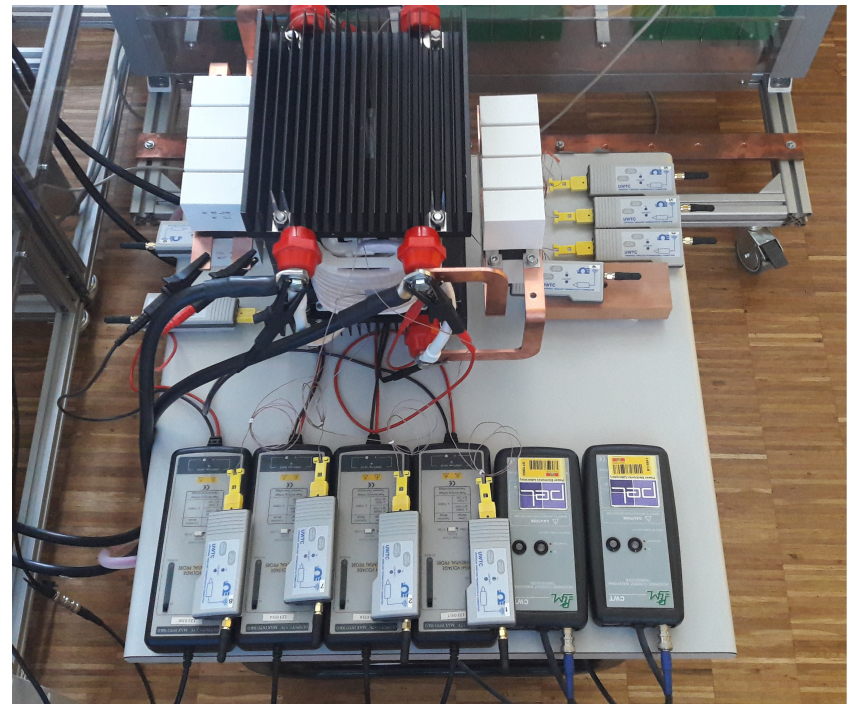

Fig. 15. MFT equipped with thermocouple probes for hot-spot temperature measurement

lated from the voltage and current readings on the two DC power supplies $U_{D C 1}$ and $I_{D C}$. The total power injected into the setup at the nominal power was measured as $2.9 \mathrm{~kW}$, indicating the overall resonant converter efficiency of $97.1 \%$. This is a reasonable result, taking into account that, with the exception of the MFT, the setup was not optimized for the given operation. On the other hand, it is hard to accurately separate the losses using data from the conducted tests, thus 


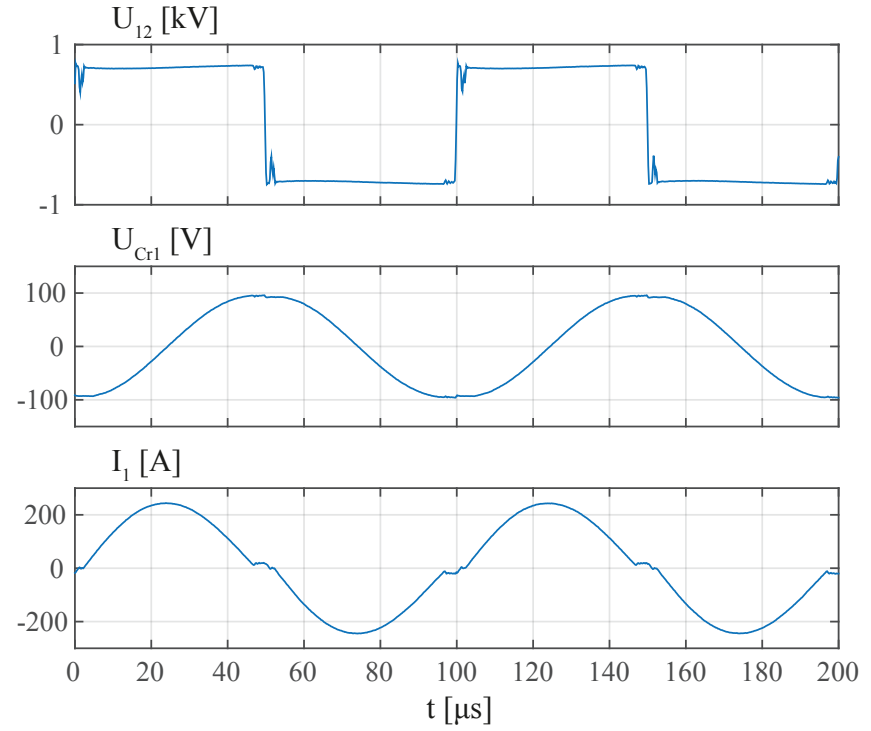

(a)
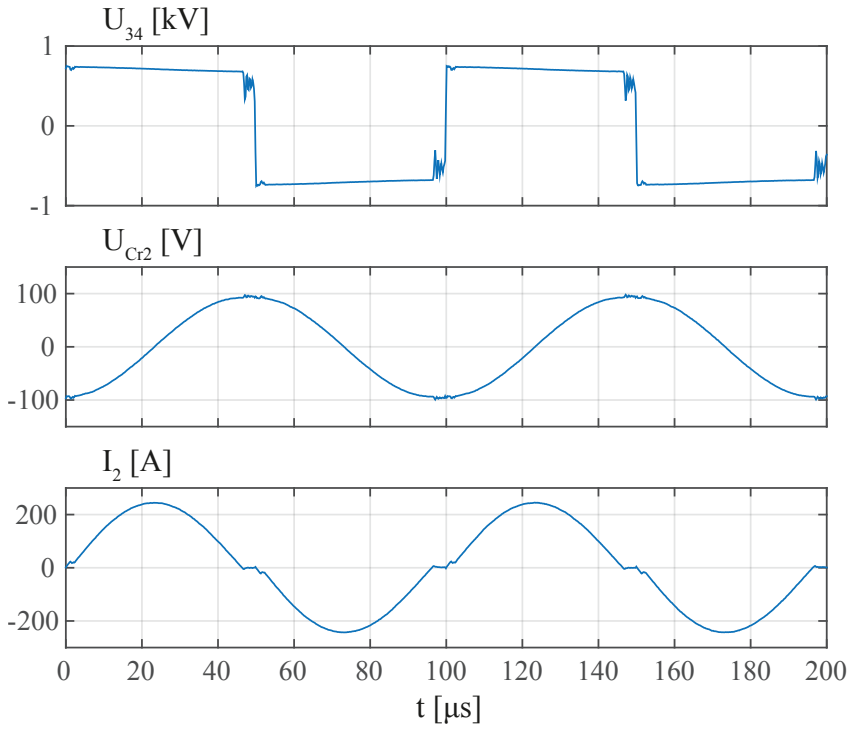

(b)

Fig. 16. Measured waveforms on the primary (a) and secondary (b) MFT side at nominal power: voltages on the inputs of the resonant tank (top), voltages on the resonant capacitors (middle) and resonant currents (bottom)

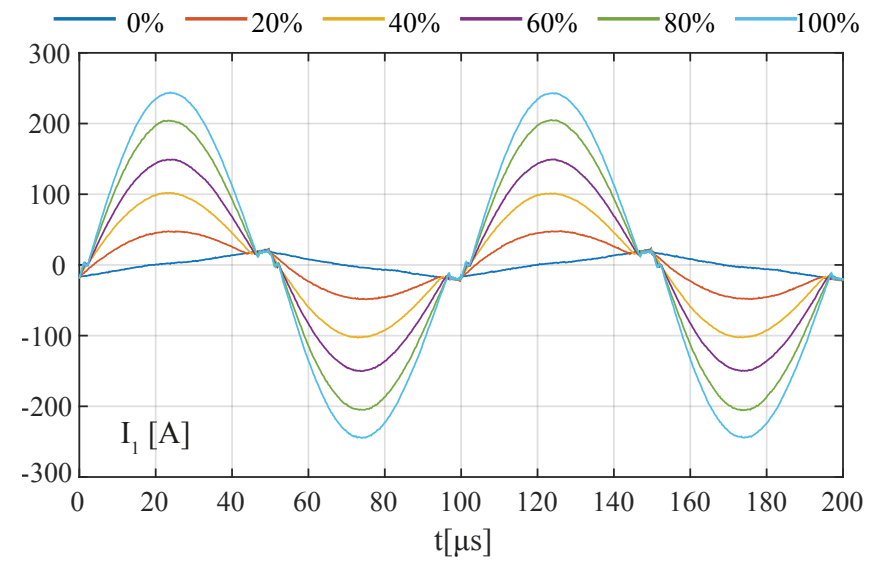

(a)

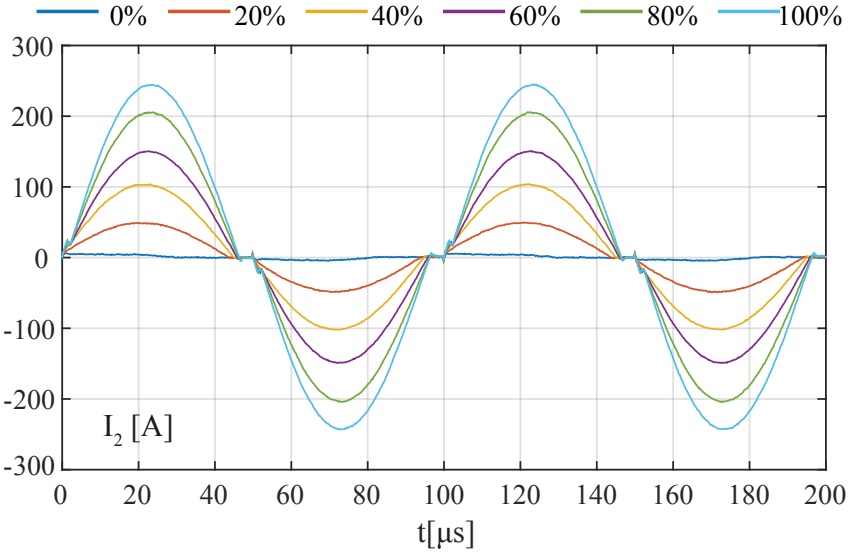

(b)

Fig. 17. Measured current waveforms on the primary (a) and secondary (b) side of the MFT at different loading conditions in range from $0 \%$ to $100 \%$ of the rated power

making the exact estimation of the MFT efficiency difficult. However, by calculating the semiconductor losses in the setup, considering well defined operating conditions, they account to $2.2 \mathrm{~kW}$ (this does not include DC link and resonant capacitor losses). Thus, the MFT efficiency can be estimated to be above $99.3 \%$ at rated operating conditions.

\section{Thermal Measurements}

To verify the thermal characteristic of the MFT, a nine hour thermal run was performed, with results presented in Fig. 18. The MFT is first operating at no load, with nominal voltage excitation until the steady state is reached. As characteristic for this operation where only the core losses are present, it can be seen that the core has the highest temperature, whereas the windings are predominantly heated through the coupling
(Fig. 5b), considering low primary winding magnetization current, as already presented in Fig. 17.

Following prolonged no load operation, the MFT is loaded with the nominal power, where both core and winding losses are at their maximal value. It can be seen that the winding temperature is now the highest, in compliance with thermal design expectation, as given in Table II. The measured core and secondary winding temperatures are slightly higher than estimated, but still within the safe operating limits. Given the good correlation between FEM and analytical model estimations, it can be concluded that this discrepancy is mainly due to the low accuracy of the applied empiric natural convection formulas. Thermal camera images, depicting the MFT surface temperatures at the two described steady states, are given in Fig. 19. 
Cooler Central Point Temperature $\left[{ }^{\circ} \mathrm{C}\right]$
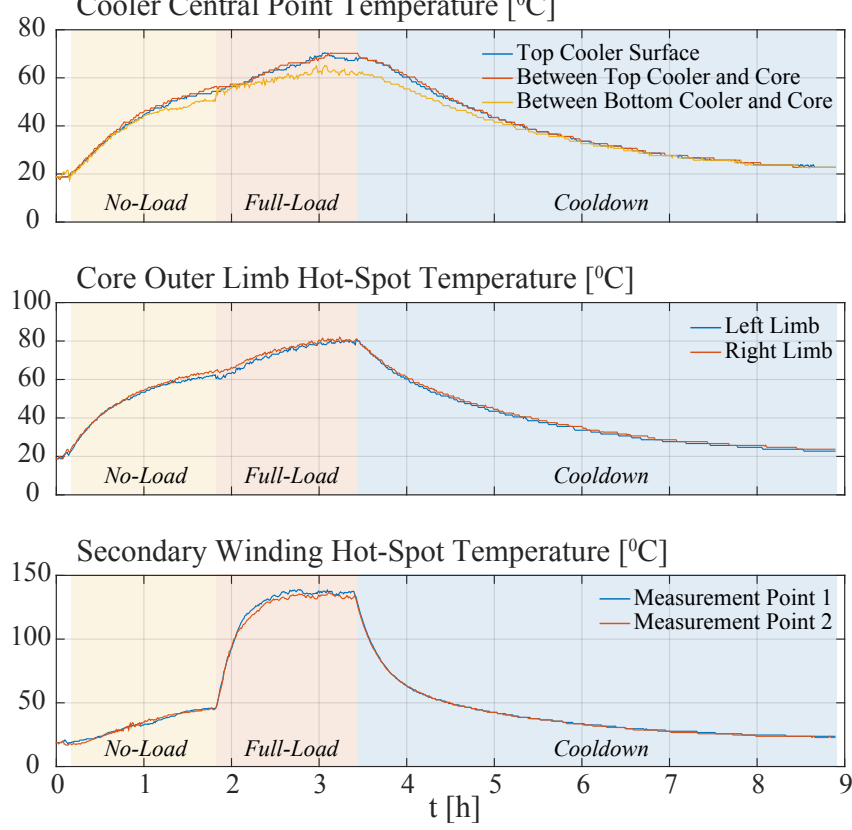

Fig. 18. Plots of the thermocouple probe measurements during the ninehour thermal run. Measurement points 1 and 2 correspond to hot-spots of the portions of the winding inside and outside of the core window area, respectively.

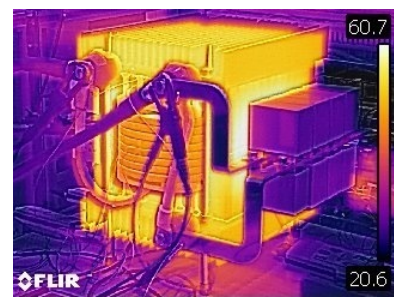

(a)

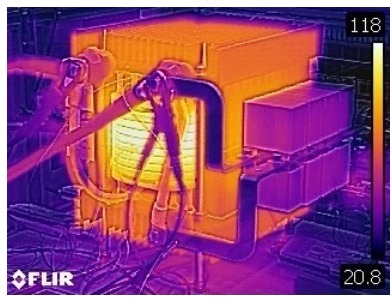

(b)
Fig. 19. Thermal camera images of the MFT: (a) in no-load steady state excited with nominal voltage input from primary side; (b) in full-load steady state;

\section{Dielectric Withstand Test}

Finally, to verify the achieved voltage insulation levels, the MFT prototype was tested in a high voltage test setup (able to generate voltages up to $100 \mathrm{kV}$ ) displayed in Fig. 20, normally used for AC dielectric withstand and partial discharge tests. The tests were carried by applying the voltage: i) between the primary and the secondary winding; ii) between the secondary winding and the conductive parts of the MFT (top and the bottom heat-sinks and the vertical studs). The voltage profile has been set considering recommendation outlined in safety standards (e.g. IEC 60664-1). The critical partial discharge level $(10 \mathrm{pC})$ has been recorded at $4 \mathrm{kV} \mathrm{AC}$, applied between the primary and the secondary windings, with measurement results shown in Fig. 21 (obtained with Omicron MPD600 system).

\section{CONCLUSIONS}

A complete, brute force based, MFT design optimization process, relying on detailed analytical modeling has been

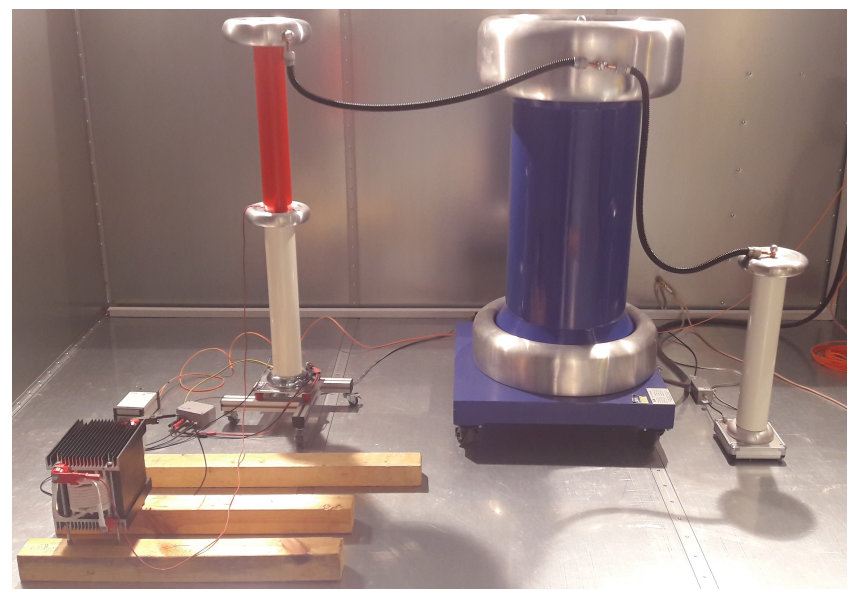

Fig. 20. MFT dielectric withstand test

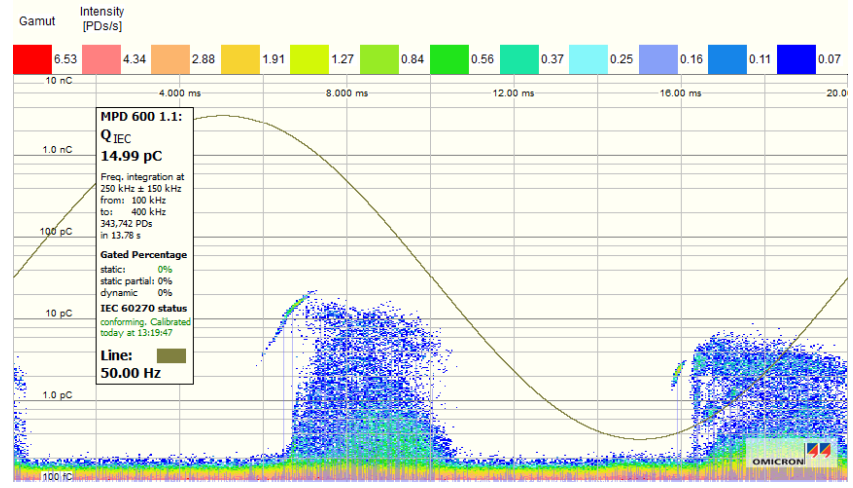

Fig. 21. Plot recorded during the partial discharge testing between primary and secondary winding for a standard PD test profile (front $V=6 \mathrm{kV}$ and flat back $V=4 \mathrm{kV}$ )

presented and experimentally verified. The results of the application of the proposed design procedure, on a specific MFT design specifications, have been illustrated and analyzed in detail, exposing various design trade-offs that must be carefully considered. While the design tool can develop, analyze and compare large number of designs (e.g. several hundred of thousands to couple of millions), ultimate selection of a design is left to the user, who is greatly assisted with developed filters, allowing easy and intuitive navigation through a design space.

To verify the utilized modeling and effectiveness of the design process, a $100 \mathrm{~kW}, 10 \mathrm{kHz}$ rated MFT prototype has been realized using the standard off-the-shelf components, based on the design generated by the algorithm. Despite the best effort by the authors, mechanical imperfections and limitations of the integration process, have resulted in slight deviation of some electrical parameters, providing useful feedback for improvements of robustness of the optimization process as well as relevant analytical models.

Realized MFT prototype has been successfully tested at various operating resonant conditions in a full power rated resonant converter. Test results have confirmed targeted electrical performance which are in accordance with design specifications, achieving high efficiency without any thermal issues. Developed design optimization procedure was successfully verified, proving its usefulness and providing assurance for 
future designs.

\section{REFERENCES}

[1] J. E. Huber and J. W. Kolar, "Volume/weight/cost comparison of a 1MVA $10 \mathrm{kV} / 400 \mathrm{~V}$ solid-state against a conventional low-frequency distribution transformer," in 2014 IEEE Energy Conversion Congress and Exposition (ECCE), Sep. 2014, pp. 4545-4552.

[2] D. Dujic, F. Kieferndorf, F. Canales, and U. Drofenik, "Power electronic traction transformer technology," in Power Electronics and Motion Control Conference (IPEMC), 2012 7th International, vol. 1, Jun. 2012, pp. 636-642.

[3] D. Dujic, C. Zhao, A. Mester, J. K. Steinke, M. Weiss, S. Lewdeni-Schmid, T. Chaudhuri, and P. Stefanutti, "Power Electronic Traction Transformer-Low Voltage Prototype," IEEE Transactions on Power Electronics, vol. 28, no. 12, pp. 5522-5534, Dec. 2013.

[4] C. Zhao, D. Dujic, A. Mester, J. K. Steinke, M. Weiss, S. Lewdeni-Schmid, T. Chaudhuri, and P. Stefanutti, "Power electronic traction transformer-medium voltage prototype," IEEE Transactions on Industrial Electronics, vol. 61, no. 7, pp. 3257-3268, Jul. 2014.

[5] D. Wang, J. Tian, C. Mao, J. Lu, Y. Duan, J. Qiu, and H. Cai, "A 10-kv/400-v 500-kva electronic power transformer," IEEE Transactions on Industrial Electronics, vol. 63, no. 11, pp. 6653-6663, Nov. 2016.

[6] K. Venkatachalam, C. Sullivan, T. Abdallah, and H. Tacca, "Accurate prediction of ferrite core loss with nonsinusoidal waveforms using only Steinmetz parameters," in Proc. of IEEE Workshop on Computers in Power Electronics, Jun. 2002, pp. 36-41.

[7] J. Reinert, A. Brockmeyer, and R. De Doncker, "Calculation of losses in ferro- and ferrimagnetic materials based on the modified Steinmetz equation," IEEE Transactions on Industry Applications, vol. 37, no. 4, pp. 1055-1061, Jul. 2001.

[8] D. Lin, P. Zhou, W. Fu, Z. Badics, and Z. Cendes, "A dynamic core loss model for soft ferromagnetic and power ferrite materials in transient finite element analysis," IEEE Transactions on Magnetics, vol. 40, no. 2, pp. 1318-1321, Mar. 2004.

[9] C.R. Sullivan, "Optimal choice for number of strands in a litz-wire transformer winding," IEEE Transactions on Power Electronics, vol. 14, no. 2, pp. 283-291, Mar. 1999.

[10] R. W. Erickson and D. Maksimovic, Fundamentals of Power Electronics, en. Springer US, Jan. 2001.

[11] F. Tourkhani and P. Viarouge, "Accurate analytical model of winding losses in round Litz wire windings," IEEE Transactions on Magnetics, vol. 37, no. 1, pp. 538-543, Jan. 2001.

[12] G. S. Dimitrakakis, E. C. Tatakis, and E. J. Rikos, "A new model for the determination of copper losses in transformer windings with arbitrary conductor distribution under high frequency sinusoidal excitation," in 2007 European Conference on Power Electronics and Applications, Sep. 2007, pp. 1-10.
[13] A. Stadler, "The optimization of high frequency inductors with litz-wire windings," in 2013 8th International Conference on Compatibility and Power Electronics (CPE), Jun. 2013, pp. 209-213.

[14] J.G. Breslin and W.G. Hurley, "Computer aided high frequency transformer design using an optimized methodology," in The 7th Workshop on Computers in Power Electronics, 2000. COMPEL 2000, 2000, pp. 277-280.

[15] W.G. Hurley, E. Gath, and J. Breslin, "Optimizing the AC resistance of multilayer transformer windings with arbitrary current waveforms," IEEE Transactions on Power Electronics, vol. 15, no. 2, pp. 369-376, Mar. 2000.

[16] P. Thummala, H. Schneider, Z. Zhang, Z. Ouyang, A. Knott, and M. A. E. Andersen, "Efficiency Optimization by Considering the High-Voltage Flyback Transformer Parasitics Using an Automatic Winding Layout Technique," IEEE Transactions on Power Electronics, vol. 30, no. 10, pp. 5755-5768, Oct. 2015.

[17] C. R. Sullivan, T. Abdallah, and T. Fujiwara, "Optimization of a flyback transformer winding considering twodimensional field effects, cost and loss," in Sixteenth Annual IEEE Applied Power Electronics Conference and Exposition, 2001. APEC 2001, vol. 1, 2001, 116-122 vol.1.

[18] J. G. Breslin and W. G. Hurley, "Derivation of optimum winding thickness for duty cycle modulated current waveshapes," in , 28th Annual IEEE Power Electronics Specialists Conference, 1997. PESC '97 Record, vol. 1, Jun. 1997, 655-661 vol.1.

[19] R. P. Wojda and M. K. Kazimierczuk, "Winding resistance of litz-wire and multi-strand inductors," IET Power Electronics, vol. 5, no. 2, pp. 257-268, Feb. 2012.

[20] M. Mogorovic and D. Dujic, "Medium frequency transformer leakage inductance modeling and experimental verification," in IEEE Energy Conversion Congress and Exposition (ECCE) 2017, Cincinnatti, OH, USA, 2017., pp. 419-424.

[21] , "Thermal modeling and experimental verification of an air cooled medium frequency transformer," in 19th European Conference on Power Electronics and Applications (EPE'17 ECCE Europe), Warsaw, Poland, 2017., pp. 1-10.

[22] I. Villar, "Multiphysical Characterization of MediumFrequency Power Electronic Transformers," PhD thesis, EPFL Lausanne, Switzerland, 2010.

[23] G. Ortiz, "High-Power DC-DC Converter Technologies for Smart Grid and Traction Applications," PhD thesis, ETH Zurich, Switzerland, 2014.

[24] M. Bahmani, "Design and Optimization Considerations of Medium-Frequency Power Transformers in HighPower DC-DC Applications," PhD thesis, Chalmers University of Technology Gothenburg, Sweden, 2016.

[25] P. L. Dowell, "Effects of eddy currents in transformer windings," Proc. of the Institution of Electrical Engineers, vol. 113, no. 8, pp. 1387-1394, Aug. 1966. 
[26] S. V. Kulkarni and S. A. Khaparde, Transformer Engineering: Design and Practice. Marcel Dekker, Inc., 2004.

[27] C. W. T. McLyman, Designing magnetic components for high frequency DC-DC converters, en. KG Magnetics, Feb. 1993.

[28] A. Van den Bossche and V. C. Valchev, Inductors and Transformers for Power Electronics. Taylor \& Francis, Mar. 2005.

[29] Convection From a Rectangular Plate. http://people.csail.mit.edu/jaffer/SimRoof/Convection/.

[30] F. M. White, Viscous Fluid Flow. McGraw-Hill Higher Education, 2006.

[31] F. Dorfler and F. Bullo, "Kron reduction of graphs with applications to electrical networks," IEEE Transactions on Circuits and Systems I: Regular Papers, vol. 60, no. 1, pp. 150-163, Jan. 2013.

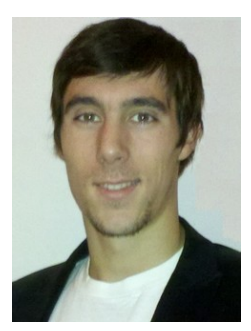

Marko Mogorovic Marko Mogorovic (M'15) received the B.S. degree in electrical engineering from University of Belgrade, Belgrade, Serbia, in 2013 and M.S. degree in smart grid science and technology at École Polytechnique Fédérale de Lausanne (EPFL), Lausanne, Switzerland, in 2015. During his M.S., he spent one year at ABB Medium Voltage Drives, Turgi, Switzerland as a researcher. In 2015 he joined Power Electronics Laboratory of École Polytechnique Fédérale de Lausanne (EPFL) in Lausanne, Switzerland as a doctoral research assistant, pursuing his $\mathrm{PhD}$ degree. His current research interests include modeling, design and optimization of galvanically isolated high power medium voltage converters.

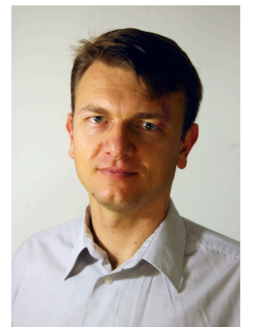

Drazen Dujic Drazen Dujic (S'03-M'09-SM'12) received the Dipl.-Ing. and M.Sc. degrees from the University of Novi Sad, Novi Sad, Serbia, in 2002 and 2005, respectively, and the Ph.D. degree from the Liverpool John Moores University, Liverpool, U.K., in 2008, all in electrical engineering. From 2002 to 2006, he was with the Department of Electrical Engineering, University of Novi Sad as a Research Assistant, and from 2006 to 2009 with Liverpool John Moores University as a Research Associate. From 2009 till 2013, he was with ABB Corporate Research Centre, Switzerland, as a Principal Scientist working on the power electronics projects spanning the range from low-voltage/power SMPS in below kilowatt range to medium voltage high-power converters in a megawatt range. During 2010-2011, he was a member of a project team responsible for the development of the worlds first power electronic traction transformer (PETT) successfully commissioned on the locomotive. From 2013 till 2014, he was with ABB Medium Voltage Drives, Turgi, Switzerland, as R\&D Platform Manager, responsible for ABB's largest IGCT based medium voltage drive - ACS6000. He is currently with Ecole Polytechnique Federale de Lausanne EPFL, Lausanne, Switzerland, as an Assistant Professor and the Director of the Power Electronics Laboratory. His current research interests include the areas of design and control of advanced high-power electronics systems and high performance drives. He has authored or coauthored more than 100 scientific publications and has filed twelve patents. He is an Associate Editor for IEEE Transactions on Industrial Electronics, IEEE Transaction on Power Electronics and IET Electric Power Applications. He has received the First Prize Paper Award by the Electric Machines Committee of the IEEE Industrial Electronics Society at IECON-2007. In 2014 he has received the Isao Takahashi Power Electronics Award for outstanding achievement in power electronics. 